\title{
A pathway level analysis of PFAS exposure and risk of gestational diabetes mellitus
}

\author{
Rahel L. Birru', Hai-Wei Liang ${ }^{1}$, Fouzia Farooq ${ }^{1}$, Megha Bedi², Maisa Feghali³, Catherine L. Haggerty', \\ Dara D. Mendez ${ }^{1}$, Janet M. Catov ${ }^{1,3}$, Carla A. $\mathrm{Ng}^{2,4}$ and Jennifer J. Adibi ${ }^{1,3^{*}}$ (D)
}

\begin{abstract}
Per- and polyfluoroalkyl substances (PFAS) have been found to be associated with gestational diabetes mellitus (GDM) development, a maternal health disorder in pregnancy with negative effects that can extend beyond pregnancy. Studies that report on this association are difficult to summarize due to weak associations and wide confidence intervals. One way to advance this field is to sharpen the biologic theory on a causal pathway behind this association, and to measure it directly by way of molecular biomarkers. The aim of this review is to summarize the literature that supports a novel pathway between PFAS exposure and GDM development. Epidemiological studies demonstrate a clear association of biomarkers of thyroid hormones and glucose metabolism with GDM development. We report biologic plausibility and epidemiologic evidence that PFAS dysregulation of maternal thyroid hormones and thyrotropin (TSH) may disrupt glucose homeostasis, increasing the risk of GDM. Overall, epidemiological studies demonstrate that PFAS were positively associated with TSH and negatively with triiodothyronine (T3) and thyroxine (T4). PFAS were generally positively associated with glucose and insulin levels in pregnancy. We propose dysregulation of thyroid function and glucose metabolism may be a critical and missing component in the accurate estimation of PFAS on the risk of GDM.
\end{abstract}

Keywords: PFAS, Gestational diabetes mellitus, Thyroid hormones, Glucose metabolism

\section{Introduction}

Per- and polyfluoroalkyl substances (PFAS) are synthetic organic chemicals used ubiquitously in numerous industries that contaminate food, water, and air, resulting in human exposure. Some PFAS can be bioaccumulative and toxic. Elevated prenatal PFAS levels have been associated with maternal health disorders, including gestational diabetes mellitus (GDM). GDM is an endocrine disorder that develops during pregnancy and can have lasting impacts on maternal health, partly through conversion to type II diabetes

\footnotetext{
* Correspondence: adibij@pitt.edu

${ }^{1}$ Department of Epidemiology, Graduate School of Public Health, University of Pittsburgh, Pittsburgh, PA, USA

${ }^{3}$ Department of Obstetrics, Gynecology, and Reproductive Sciences,

University of Pittsburgh School of Medicine, Pittsburgh, PA, USA

Full list of author information is available at the end of the article
}

(T2D). The mechanism of GDM development is poorly understood and generally not addressed in epidemiologic investigations on PFAS exposures and their impacts. We review the literature for candidate biomarkers of GDM, specifically highlighting those measuring maternal thyroid and maternal glucose homeostasis. We then review the epidemiologic literature for evidence of PFAS as a putative cause of GDM by way of molecular biomarkers in a pathway that connects PFAS toxicity to GDM. We propose that a systematic approach to examining this pathway through validated biomarker measurement can improve the understanding of the pathophysiology of GDM. Additionally, we suspect that this approach will reveal at which points PFAS have their strongest effects. 


\section{Introduction to perfluoroalkyl and polyfluoroalkyl substances (PFAS)}

PFAS are a family of synthetic chemicals diverse in size and structure that contain at least one perfluoroalkyl moiety $\left(\mathrm{C}_{n} \mathrm{~F}_{2 n+1^{-}}\right)$. Frequently studied PFAS include perfluorooctane sulfonate (PFOS), perfluorooctanoic acid (PFOA), perfluorononanoic acid (PFNA), perfluorodecanoic acid (PFDA), perfluoroundecanoic acid (PFUnDA), and perfluorohexane sulfonic acid (PFHxS). PFAS have both hydrophobic and oleophobic characteristics that are used in commercial products to increase stain repellency and non-stick qualities, food processing equipment and packaging to reduce oil transfer, firefighting activities as a component of aqueous film forming foams, and industrial and manufacturing practices for purposes such as liquid cooling $[1,2]$. Due to their strong carbon-fluorine bonds, they are resistant to degradation [1]. Regulations have been enacted in many countries to eliminate the most common PFAS, mainly PFOS and PFOA; yet they continue to circulate after production stops due to their ability to persist and accumulate in the environment [1]. Furthermore, new short-chain perfluoroalkyl substances and other structurally diverse PFAS developed by industries as replacements to PFOA and PFOS are increasingly being found to be equally toxic [1]. Therefore, understanding the biological effects of this class of chemicals continues to be a priority.

In a National Health and Nutrition Examination Survey (NHANES) study of 7876 US participants $\geq 12$ years old, four of the 12 PFAS measured (PFOS, PFOA, PFNA, and PFHxS) were detected in 95\% of serum samples [3]. Although concentrations of some PFAS, like PFOS, generally declined over the period of 1999 to 2008, others remained constant or increased, including PFOA and PFNA [3]. PFAS levels are highly prevalent globally and measured consistently in US and non-US cohorts [4]. The average half-life of PFAS in the human body varies, with long-chain compounds having increased half-lives and ability to bioaccumulate $[1,5]$. Serum half-life measurements have mainly been reported for a selection of long-chain PFAS (PFOS, PFOA, and PFHxS), with a range from 3 to 5 years $[1,6]$. $\mathrm{Xu}$ et al. found that these legacy PFAS had half-lives of approximately 2 to 3 years in a Swedish cohort and accounted for $90 \%$ of the total serum PFAS compared to short-chain PFAS (i.e. perfluorobutane sulfonic acid, perfluoroheptanoic acid), which had half-lives of less than 1.5 years and lower bioavailability [7]. All PFAS, however, have relatively long environmental half-lives due to minimal degradation and can remain bioavailable to humans over long periods of time through exposures such as drinking water [1].

\section{PFAS impacts on maternal health and pregnancy}

Acute hormonal and immune changes underway during pregnancy render women and their fetuses particularly vulnerable to PFAS exposure. As women drink more during pregnancy, water may be a significant exposure source. PFAS plasma levels in pregnant women in Shanghai, a particularly contaminated region, were higher in those drinking tap water compared to purified or bottled water, independent of diet [8]. There is epidemiological evidence that PFAS affects maternal health during pregnancy, including contributing to GDM development. Clinically, GDM is diagnosed as type I diabetes (T1D) or T2D presenting in the second or third trimester of pregnancy that is not preexisting [9]. GDM prevalence in 2015 was $8.8 \%$ of pregnancies resulting in live births worldwide [10]. GDM increases the risk of low or high birth weight, preterm birth, and preeclampsia [9]. GDM can also have a long-lasting negative impact on maternal health through its conversion into T2D in approximately $50 \%$ or more of women [11] and by initiating vascular changes that increase cardiovascular disease risk [9].

Even though associations of PFAS with diabetes and GDM risk have been reported, there has been minimal work in outlining a detailed and plausible biological mechanism that starts with PFAS and ends with glucose dysfunction that is a proximal cause of GDM [1]. Without a theory on the endocrine mechanism that links this exposure and outcome, associations are difficult to interpret. We narrow the pathway described here to include thyroid hormones (THs) in the maintenance of glucose homeostasis in pregnant and non-pregnant populations. We hypothesize that this is a primary, but not exclusive, pathway relevant to this exposure and outcome. PFAS can disrupt the function of the maternal thyroid, which is a master regulator of glucose homeostasis. Proper thyroid functioning is a known determinant of successful pregnancy outcomes and fetal development, therefore pregnant women are particularly at risk of PFAS thyrotoxicity. This review examines epidemiologic research on circulating biomarkers of thyroid function (hormones, thyroid autoantibodies), glucose metabolism (blood glucose, glucose tolerance), and insulin secretion (insulin resistance) to offer mechanistic insight into subclinical and clinical GDM (Fig. 1). We propose a biologic pathway where PFAS disruption of maternal thyroid function alters circulating $\mathrm{TH}$ concentrations and downstream glucose homeostasis, resulting in an increased risk for GDM development.

PFAS encompass thousands of compounds that may elicit different biological responses, particularly new and emerging compounds whose toxicities have yet to be examined. The majority of these compounds, however, have not been studied in relation to the proposed 


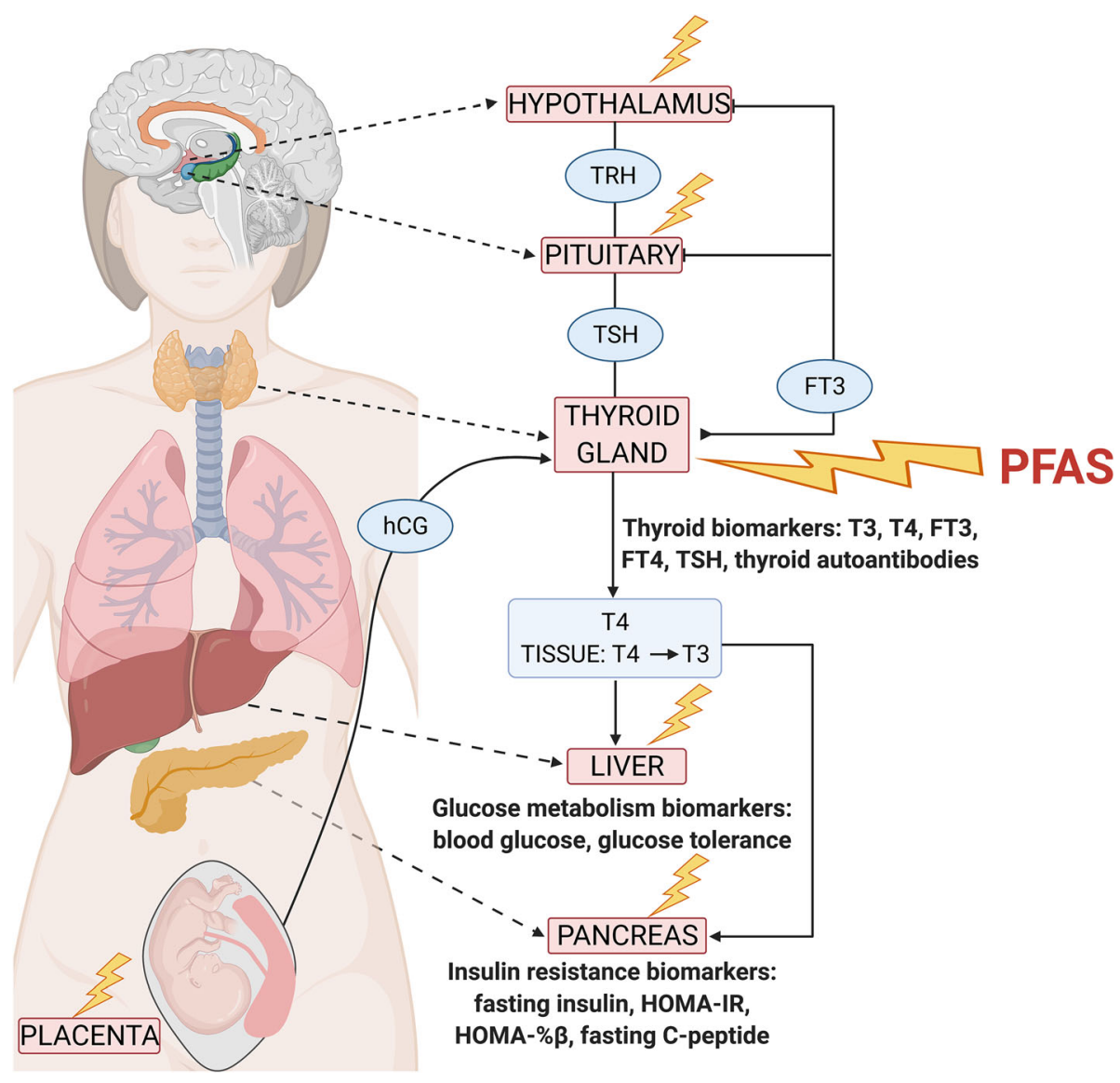

Fig. 1 Novel biomarkers in the pathway between PFAS exposure and gestational diabetes mellitus (GDM) development. PFAS targets the hypothalamic-pituitary-thyroid axis, disrupting thyroid hormone homeostasis, which may contribute to GDM development. While the thyroid may be the main target for hormone disruption, PFAS can also exert toxicity to other tissues involved in this regulatory pathway. Abbreviations: FT3, free triiodothyronine; FT4, free thyroxine; $h C G$, human chorionic gonadotropin; HOMA-\% $\beta$, homeostatic model assessments of $\beta$-cell function; HOMA-IR, homeostatic model of assessment of insulin resistance; T3, triiodothyronine; T4, thyroxine; TRH, thyrotropin-releasing hormone; TSH, thyrotropin

pathway. Therefore, this review is limited to PFAS with the highest circulating concentrations ubiquitously measured in the population and most commonly studied in the literature (Supplemental Table 2), mainly PFOA, PFOS, PFNA, PFDA, PFUnDA, and PFHxS, though there are many PFAS which are not toxic or biologically active in the same way.

\section{Thyroid hormones regulate normal maternal glucose homeostasis in non-pregnant and pregnant women}

The thyroid gland, by way of its hormones, regulates multiple developmental and metabolic pathways involved in energy storage and expenditure, cardiovascular function, and glucose metabolism [12]. TH secretion is controlled by the hypothalamic-pituitary-thyroid axis (Fig. 1) [12, 13]. Briefly, thyrotropin-releasing hormone (TRH) is released by the hypothalamus, which stimulates the release of thyrotropin (TSH) by the pituitary gland $[12,13]$. TSH binds to its receptor on the thyroid gland, which results in the production and release of the THs triiodothyronine (T3) and thyroxine (T4) [12]. Circulating THs can be in their biologically active unbound forms free T3 (FT3) or free T4 (FT4) but are primarily bound to transport proteins (over 99\%) that facilitate their movement and binding to their receptors across the body $[12,13]$. Circulating THs inhibit TRH and TSH release, completing the normal negative feedback loop [12]. Types 1, 2, and 3 iodothyronine deiodinases (D1, D2, and D3, respectively) catalyze the conversion of THs into their biologically active or inactive forms [13]. While circulating $\mathrm{TH}$ levels are typically held constant at the 'global level' by the hypothalamic-pituitary-thyroid axis, tissue-specific expression and activity of TH receptors, transporters, and deiodinases can modulate $\mathrm{TH}$ concentrations to allow for local regulation of $\mathrm{TH}$ controlled processes $[14,15]$.

Glucose production and storage by the liver is one example of tissue-specific TH action. THs regulate glucose 
levels by increasing glucose production in the liver. On a transcriptional level, THs regulate several liver enzymes in the gluconeogenesis and glycogenolysis pathways to increase glucose levels, including phosphoenolpyruvate carboxykinase and glucose-6-phosphatase [12, 16, 17]. Additionally, THs regulate glucose transporters. THs increase expression of the hepatic glucose transporter GLUT2, increasing glucose output $[17,18]$. T3 increases GLUT4 gene expression in all cells, which encodes the transporter responsible for the insulin-mediated uptake of glucose [12, 17].

THs also modulate glucose homeostasis by regulating the differentiation and function of the islet cells of the maternal pancreas. Pancreatic islet cells, which produce insulin, express $\mathrm{TH}$ receptors $[12,15]$. Insulin signals for all cell types in the body to take up glucose and store it as glycogen to maintain physiologically appropriate levels of glucose in the blood. The binding of T3 to the islet transcription factor MAFA results in the maturation of islet cells to become glucose-responsive and insulinsecreting $[12,15]$.

Disorders in the adult thyroid can disrupt blood glucose control. Hyperthyroidism is defined as low concentrations of TSH and elevated FT3 and/or FT4 concentrations [19]. The thyroid produces excess THs, increasing gluconeogenesis and resulting in elevated blood glucose levels $[13,15]$ and hepatic insulin resistance [17]. High doses of THs can also impair islet function in the pancreas, resulting in reduced insulin secretion and subsequent glucose intolerance [15]. Hence, hyperthyroidism is a cause of diabetes mellitus both through glucose overproduction and by inhibition of glucose uptake by the cells $[13,15]$. Hypothyroidism is clinically defined as elevated TSH and low FT4 concentrations [19] and has also been associated with diabetes mellitus. In this disorder, liver gluconeogenesis is inhibited [17]. The effects of hypothyroidism include altered phosphorylation of insulin signaling proteins, dysregulation of leptin in the hypothalamus, and impaired adipose and skeletal muscle function (such as diminished blood flow and muscle oxidative capacity) [17]. The net result of these changes is increased insulin resistance in the peripheral tissues, resulting in a reduction in glucose utilization and altered glucose homeostasis $[17,18]$.

Autoimmune thyroid diseases (AITDs) are a main cause of toxicity to the thyroid system and therefore may also indirectly contribute to glucose homeostasis disruption. AITDs are the most common causes of hyperthyroidism and hypothyroidism [20]. These include Graves' disease (GD), associated with hyperthyroidism, and Hashimoto's thyroiditis (HT), associated with hypothyroidism. Therefore, markers of AITDs can also be considered as biomarkers for thyroid function. The presence of autoantibodies, namely thyroid peroxidase antibody (TPOAb) and thyroglobulin antibody (TgAb), have been measured in individuals who develop autoimmunity [21]. TPOAb targets the thyroid peroxidase (TPO) enzyme, which is responsible for adding iodine to thyroglobulin $(\mathrm{Tg})$, the precursor of $\mathrm{T} 3$ and $\mathrm{T} 4$ [13, 22] and TgAb targets Tg [22]. Hence, elevation of either or both TPOAb and TgAb are indicators of potential toxicity to the system. While genetic factors account for approximately $70 \%$ of AITDs, environmental toxicant exposures, including PFAS, are also associated with elevated thyroid autoimmunity biomarker levels [23].

\section{Thyroid and glucose regulation by the fetal placenta during pregnancy}

The fetal placenta produces a variety of hormones that enter maternal circulation and stimulate maternal hormone production, including THs [24]. In the first 10 weeks of pregnancy, there is a steady increase in human chorionic gonadotropin (hCG) produced by placental trophoblasts [24]. In the period in which hCG reaches its peak physiological concentrations (3-10 weeks), hCG can bind to the maternal thyroid TSH receptor. Due to its similar structure to TSH, hCG can stimulate TH release, particularly FT4 $[24,25]$. The transport protein TBG also rises during this time alongside hCG, stimulated by estrogen, increasing total T4 levels [25]. TSH release by the pituitary gland is suppressed by rising THs and hCG due to the negative feedback relationship $[25,26]$. Placental factors other than hCG, such as placental growth factor (PLGF), may also regulate the normal function of maternal thyroid [27, 28].

The fetal thyroid gland does not begins its own hormone production until 16 weeks, yet the fetus requires THs to regulate multiple processes before this point, including successful embryonic implantation and fetal neurodevelopment $[29,30]$. The fetus is fully dependent on maternal TH production in the first trimester [31, 32]. Insufficient first trimester maternal $\mathrm{TH}$ levels are associated with pregnancy loss and impaired neuropsychomotor development of the child [30,33, 34]. Transplacental transfer of maternal THs into the fetal compartment is facilitated by $\mathrm{TH}$ binding proteins and transporters in the placenta $[31,35]$. Deiodinase enzymes in the placenta (D2, D3) regulate fetal exposure to maternal $\mathrm{THs}$, either by metabolizing T4 into the bioactive $\mathrm{T} 3$ or inactivating the hormones [35].

In a study of 18,683 pregnant women, women at the 85th-95th percentile of the first trimester hCG multiple of the median (MoM, a measure of hCG that is normalized for gestational age) had $6.4-11.8 \%$ lower risk of GDM incidence, respectively [36]. In this study, FT4 mediated $21.4 \%$ of the hCG-GDM association [36]. The placenta may be an important mediator in the regulation of glucose homeostasis by the maternal thyroid and subsequent risk of GDM. Higher first trimester hCG was 
associated with a reduced risk of GDM development and negatively associated with blood glucose levels [36, 37]. As gestational age advances, there is a net effect of changing maternal and placental physiology whereby maternal insulin resistance and blood glucose and free fatty acid (FFA) levels increase [9]. Glucose is transported through the placenta to the fetus to fuel growth [9]. Pancreatic $\beta$ cell hyperplasia and hypertrophy occur to stimulate more insulin production and to counteract the increasing insulin resistance [9]. After the delivery of the child and the placenta, maternal insulin sensitivity returns to pre-pregnancy levels. The placenta plays an active role in this phenomenon [9] and is under-utilized as a source of biomarkers to study maternal glucose homeostasis.

\section{Gestational diabetes mellitus (GDM): definition and candidate biomarkers}

When glucose homeostasis is highly disrupted in pregnancy due to a dysregulation of the mechanisms described above, women are at risk of developing GDM. GDM is defined as clinical hyperglycemia detected during pregnancy, secondary to insulin resistance [9]. Insulin resistance indicates that the cells are not able to properly utilize glucose leading to glucose levels in the blood that have reached a level high enough to become toxic to the cells. Risk factors such as obesity, advanced age, and family history of diabetes can lead to pancreatic $\beta$ cell dysfunction, resulting in insufficient insulin production to overcome the insulin resistance associated with pregnancy $[9,38]$. This causes blood sugar and FFA levels to increase $[9,38]$.

\section{Connecting the dots between thyroid function and gestational diabetes mellitus (GDM)}

A causal relationship between THs and the risk of GDM is also supported by epidemiologic findings. Leng et al. reported that higher first trimester TSH levels were weakly associated with increased risk of GDM in a Chinese population of 7258 women (adjusted odds ratio (OR) $1.13,95 \%$ CI 1.00, 1.27) [39]. In a longitudinal study of 321 pregnant women, FT3 and the FT3/FT4 ratio were positively associated with risk of GDM [40]. The adjusted OR of the highest versus lowest quartile of FT3 was $4.25(95 \% \mathrm{CI} 1.67,10.80)$ in the first trimester and $3.89(95 \% \mathrm{CI} 1.50,10.10)$ in the second trimester. For the FT3/FT4 ratio, the adjusted OR was $8.63(95 \%$ CI 2.87 , $26.00)$ in the first trimester and 13.60 (95\%CI 3.97 , 46.30) in the second trimester. Disruption of T3 and T4 expression as a result of hypothyroidism and hyperthyroidism is also associated with the risk of GDM [41, 42]. Some of these estimates are highly imprecise. Statistical power can be improved by increased sample size and by greater precision in the measurement of the physiologic process, which we aim to promote in this review.

Thyroid autoantibodies have also been studied as causes of GDM. In a meta-analysis, Yang et al. evaluated epidemiological studies published between 1997 and 2014 that assessed the association of thyroid autoantibodies with GDM. Among 21 studies, there was a weak association between thyroid autoantibodies and the risk of GDM (pooled relative risk (RR) 1.12, 95\%CI 1.03, 1.22), and it was comparable when women with thyroid dysfunction were included (RR 1.18, 95\%CI 1.06, 1.31) [43]. A more recent meta-analysis of thyroid dysfunction and thyroid autoimmunity by Jia et al. of 11 studies assessing thyroid function across the three trimesters of pregnancy showed a strong association between subclinical hypothyroidism, with TPOAb positivity, and GDM (OR 3.22, 95\%CI 1.72, 6.03) [44]. Similarly, evidence of an association between TPOAb and GDM was reported in a prospective study of 1683 women by Huang et al., where TPOAb positive women in early pregnancy had an increased risk of GDM (RR 2.54, 95\%CI 1.04, 6.23) [45]. These women also had significantly higher TSH and lower FT4 levels compared to euthyroid women. The presence of serum TPOAb and TgAb are indicators of higher GDM risk, making them candidate biomarkers of GDM either alone or in the context of clinically diagnosed thyroid disorders.

\section{Candidate biomarkers to examine thyroid hormone and glucose disruption in gestational diabetes mellitus (GDM) development}

GDM is diagnosed by increased values in blood glucose measurements $[38,46]$. Testing strategies in pregnancy vary and include a one-step 2-h $75 \mathrm{~g}$ oral glucose tolerance test (OGTT) or a two-step process which starts with a 1-h $50 \mathrm{~g}$ oral glucose challenge test (OGCT) followed by a diagnostic 3-h $100 \mathrm{~g}$ OGTT in women who screen positive for GDM [47]. These tests are routinely performed starting at 24-28 weeks and can serve as a source of biomarkers for use in epidemiologic studies (Fig. 1). Fasting, post-load (post consumption of a glucose-rich drink used in challenge described above), or specific glucose values can be used to assess a woman's glucose tolerance during pregnancy.

In research studies, concurrent insulin measurement can be performed to assess insulin resistance and $\beta$ cell function. A widely validated clinical and epidemiological tool is the homeostasis model assessment (HOMA), which is derived from a mathematical assessment of the balance between hepatic glucose output and insulin secretion based on fasting levels of both glucose and insulin $[46,48]$. The Matsuda index is an another method to determine insulin sensitivity from the OGTT [49]. Fasting levels of $\mathrm{C}$-peptide are indicators of pancreatic $\beta$ cell 
function. C-peptide is released by these cells during insulin secretion but it degrades slower than insulin, making it a stable biomarker of insulin secretion [50]. Based on the OGTT, a pregnant woman may be diagnosed with isolated hyperglycemia $(\mathrm{IH})$ or impaired glucose tolerance (IGT, a less mild form of glucose intolerance). The researcher can make use of her clinical diagnoses (binomial) or the continuously measured analytes such as fasting glucose or insulin levels to assess associations of THs and subclinical and clinical risk of GDM.

Candidate thyroid biomarkers to examine the causal pathway in maternal circulation include the hormones T3, T4, FT3, FT4, and TSH and thyroid autoantibodies (TPOAb and TgAb) (Fig. 1). These measures are ordered by the obstetrician commonly in the first trimester, but only in women presenting with symptoms of thyroid disease or who are at high risk (those who have an autoimmune disease such as T1D or family history of thyroid disease) [51, 52]. Small changes in FT3 and FT4 can result in a proportionally larger change in TSH due to their negative feedback relationship; therefore, TSH is the more reliable screening analyte for clinical diagnosis [51]. FT4, an accurate marker of thyroid function, is measured concurrently with TSH or in a follow-up screening to confirm a thyroid disorder diagnosis [51, 53]. Thyroid autoantibodies are also measured clinically in a small subset of women to determine women at risk of developing thyroid disorders in pregnancy, particularly those who have an autoimmune disease or have a family history of thyroid disease [51]. Measuring a full panel of THs in the research setting would allow for the most accurate understanding of thyroid function and also allow for assessment for intra- versus interindividual variability $[51,52]$.

In the last 10 years, new methods for the measurement of THs have been developed using liquid chromatography-tandem mass spectrometry (LC-MS/ MS) that have improved the sensitivity and accuracy of detection [54]. Due to the presence of TH carrier proteins in serum, free $\mathrm{TH}$ dissociation and reassociation to binding proteins can occur during sample processing, therefore altering the $\mathrm{TH}$ output measurements $[54,55]$. This is a particular problem in biospecimens derived from pregnant women, due to the spike in TBG during pregnancy $[25,54]$. Equilibrium dialysis or ultrafiltration prior to LC-MS/MS removes TH binding proteins prior to measurement of FT3 and FT4, reducing their interference [54]. LC-MS/MS allows for greater specificity compared to immunoassays by identifying analytes of interest by size rather than by antibodies. Antibodies have varied specificity to analytes and may cross-react with other metabolites in biospecimens, risking false positive results [55]. Sensitivity is enhanced because non-specific binding of antibodies and other types of interference that can mask the antibody-antigen reaction in immunoassays is avoided. This also allows for a reduced lower limit of detection of the analyte of interest [55]. LC-MS/MS methods have recently been developed for thyroid autoantibodies, but so far have comparable accuracy to the immunoassays [53].

Pregnant women are diagnosed with thyroid disease (i.e. hypothyroidism and hyperthyroidism) by comparing $\mathrm{TH}$ and antibody levels to pregnancy-specific reference ranges [52, 53]. These trimester-specific ranges account for changes in thyroid biomarker levels during pregnancy, such as the rise in FT4 and drop in TSH during the first trimester, that would otherwise appear as subclinical or clinical thyroid disease when compared to reference ranges for nonpregnant adults [52]. Universal trimester-specific ranges have been difficult to set due to differences in measurement protocols and population differences, such as iodine intake and ethnicity [52]. However, clinical laboratories can create their own reference ranges [52]. Outside of cut-offs and clinically significant $\mathrm{TH}$ levels, continuously measured $\mathrm{TH}$ biomarkers representing expression across the range of normal to abnormal variation are valuable in epidemiologic investigations to understand exposure effects and to link exposures to outcomes.

\section{PFAS exposure and risk of gestational diabetes mellitus (GDM) development and candidate biomarkers: evidence in the literature}

Epidemiologic literature was reviewed and summarized according to specific criteria (Supplement: Methods). Data were extracted and compiled in the Supplemental Tables 1-8 and summarized in Table 1. Forest plots were prepared for representative PFAS that displayed the most significant associations with selected outcomes (Figs. 2, 3). Null coefficients are not interpretable but collectively may offer insight on relationships that were underpowered but worthy of considering in future studies. Therefore, we created criteria to assess the direction of association for all outcomes moving beyond a $p$ value of less than 0.05, as described in the Methods (Supplement).

\section{PFAS and GDM}

Out of the 11 epidemiological studies that reported on the association of PFAS and GDM [56-66], four (44\%) demonstrated a positive association, differing by PFAS compound (Table 1, Supplemental Table 3) [56, 59, 62, 63]. Overall, higher PFAS levels were associated with higher risk of GDM development. Effect sizes were small and confidence intervals were wide, indicating lack of precision and/or potential bias. The strongest associations were found with PFOA, PFOS, and PFHxS, which are presented in a forest plot comparison (Fig. 2). 
Table 1 Summary of the epidemiologic studies reviewed, according to the direction of association of PFAS exposure on outcomes in the proposed pathway

\begin{tabular}{|c|c|c|c|c|}
\hline Outcome & Positive & Negative & $\begin{array}{l}\text { Null, } \\
\text { Positive Trend }\end{array}$ & $\begin{array}{l}\text { Null, } \\
\text { Negative Trend }\end{array}$ \\
\hline GDM & $36 \%$ (4/11 studies) & $0 \%$ (0/11 studies) & 91\% (10/11 studies) & $64 \%$ (7/11 studies) \\
\hline \multicolumn{5}{|l|}{ Maternal Blood } \\
\hline Glucose homeostasis biomarkers $^{\mathrm{a}}$ & 73\% (8/11 studies) & $18 \%$ (2/11 studies) & 45\% (5/11 studies) & 45\% (5/11 studies) \\
\hline T3 & $50 \%$ (2/4 studies) & $50 \%$ (2/4 studies) & $25 \%$ (1/4 studies) & $25 \%$ (1/4 studies) \\
\hline FT3 & $33 \%$ (2/6 studies) & $33 \%$ (2/6 studies) & 17\% (1/6 studies) & 17\% (1/6 studies) \\
\hline T4 & 20\% (1/5 studies) & $40 \%$ (2/5 studies) & $20 \%$ (1/5 studies) & $20 \%$ (1/5 studies) \\
\hline FT4 & $20 \%$ (2/10 studies) & $40 \%$ (4/10 studies) & $20 \%$ (2/10 studies) & $30 \%$ (3/10 studies) \\
\hline TSH & 45\% (5/11 studies) & $18 \%$ (2/11 studies) & $18 \%$ (2/11 studies) & 9\% (1/11 studies) \\
\hline
\end{tabular}

Abbreviations: GDM gestational diabetes mellitus, FT3 free triiodothyronine, FT4 free thyroxine, $T 3$ triiodothyronine, $T 4$ thyroxine, TSH thyrotropin

${ }^{a}$ Glucose homeostasis biomarkers: isolated hyperglycemia, impaired glucose tolerance, blood glucose and insulin levels, insulin resistance

${ }^{\mathrm{b} C r i t e r i a ~ d e f i n e d ~ i n ~ d e t a i l ~ i n ~ t h e ~ M e t h o d s ~(S u p p l e m e n t) ~}$

\section{PFAS exposure and biomarkers of glucose homeostasis} We next examined the association of PFAS with biomarkers of glucose homeostasis that could putatively mediate the risk of GDM. These biomarkers do not indicate frank disease but are meaningful in understanding mechanism and chronic disease risk. A two-fold increase in serum PFHxS in Danish pregnant women was positively associated with fasting glucose and insulin levels (percentage change $1.7 \%(95 \% \mathrm{CI} 0.2,3.2)$ and $7.7 \%$ (95\%CI 0.1, 15.9), respectively) [67]. Additionally, higher PFNA was associated with increased fasting insulin and $\beta$ cell function (percentage change $12.1 \%$ (95\%CI 0.7, 24.8 ) and $12.4 \%$ (95\%CI 2.2, 23.7), respectively) [67]. Eleven epidemiological studies reported on the association of PFAS with glucose and insulin biomarkers in pregnant women [56-58, 60,61, 64, 65, 67-70], with eight (73\%) finding a positive association, depending on the PFAS [56-58, 60, 64, 65, 67, 69] (Table 1, Supplemental Table 4). Only two studies (18\%) reported negative associations $[68,70]$, though levels of PFAS were lower in their study cohort compared to others examining this association (Supplemental Table 2). Additionally, the Mehta et al. study only included overweight and obese pregnant women enrolled in a stress-reduction intervention as a means to control weight gain and therefore this cohort may not reflect the general population [70]. There was evidence of nonmonotonic associations in the case of PFAS and glucose biomarkers, though these were ultimately null associations after adjustment for confounders (Supplemental Table 4). The general positive association of PFAS with glucose homeostasis biomarkers in these studies is in agreement with epidemiological studies examining PFAS exposure in non-pregnant individuals with both normal and elevated baseline glucose levels. In these populations, PFAS was found to be associated with an increased risk of diabetes and disruption of glycemic indicators, including increased blood glucose, glycated hemoglobin (HbA1c), and insulin secretion $[71,72]$.

\section{PFAS exposure and biomarkers of thyroid function}

An NHANES study of three independent study periods between 1999 and 2006 reported that among US nonpregnant adults $(n=3974)$, participants with thyroid disease had greater serum PFAS levels than those without [73]. A recent meta-analysis of 12 studies examining the effects of PFAS exposure on TH levels in adults found that PFAS are positively associated with FT4 and TSH but negatively associated with total T3 and T4 [74]. In a subgroup analysis of four studies with pregnant women within this meta-analysis $(N=1735)$, PFAS were not associated with THs [74]. Information on gestational age at the time of blood sampling for PFAS and TH measurements was not collected or controlled for; yet gestational age is a source of variability in both measures. This could be one reason for a null association.

There were 13 individual studies reviewed that measured the associations of PFAS and THs in maternal circulation [75-87], with ten (77\%) finding a positive or negative association $[75,76,79-85,87]$ (Table 1 , Supplemental Tables 5-7). Berg and colleagues published two studies examining associations of PFAS with maternal TH levels using the same cohort. Results were extracted from their 2015 study only [76, 88]. Generally, PFAS were negatively associated with THs and positively associated with TSH (Table 1). This is consistent with the idea that PFAS disrupts TH homeostasis, resulting in a reduction in $\mathrm{TH}$ levels that can stimulate TSH secretion due to the negative feedback relationship. In a systematic review examining the relationship between PFAS and THs, Ballesteros et al. also concluded that PFHxS and PFOS are generally found to be positively associated with maternal TSH in pregnancy [89]. Of the studies 


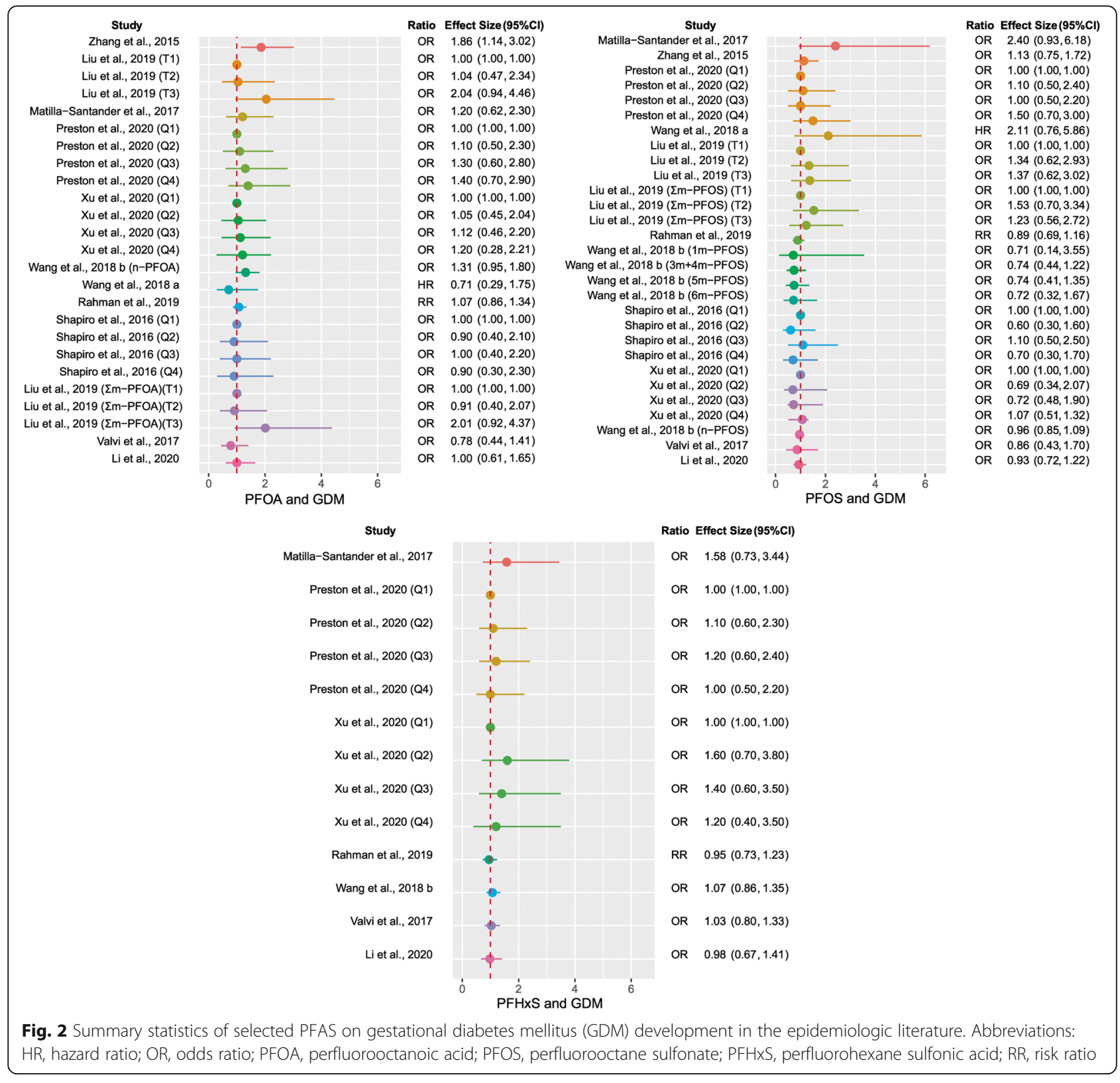

included in our review, the strongest PFAS and thyroid biomarker relationship was with TSH (Fig. 3).

Of the six studies that performed a subanalysis stratifying women by thyroid antibody status, all six reported that maternal thyroid autoimmunity altered PFAS disruption of the thyroid based on effect measure modification (Supplemental Table 8) $[75,79-82,85]$. In these studies, it is not presumed that PFAS increased autoimmunity but that PFAS may exacerbate the autoimmune damage to further dysregulate $\mathrm{TH}$ production $[79,82,85]$. Itoh et al. found that maternal PFAS levels were associated with FT3 in both maternal thyroid autoantibody positive and negative groups [79]. Consistent with studies that did not measure thyroid autoantibody status, Webster et al. found that
PFAS increased TSH and lowered FT4 levels in TPOAb positive pregnant women. Effects were milder in pregnant women with normal TPOAb levels [85]. However, in a recent US study, TPOAb positive women had a negative association between PFAS exposure and TSH levels [81], inconsistent with other studies reporting a positive association $[75,76,82,83,85]$. The authors noted that this discrepancy could be a result of study design differences, particularly iodide status of their study population, which affects TH synthesis $[81,85]$. Overall, the results indicate that thyroid autoimmunity is a key variable to consider.

Beyond disrupting maternal TH homeostasis, PFAS can be transferred from maternal circulation to the fetus to alter fetal TH levels. Several studies included in this 


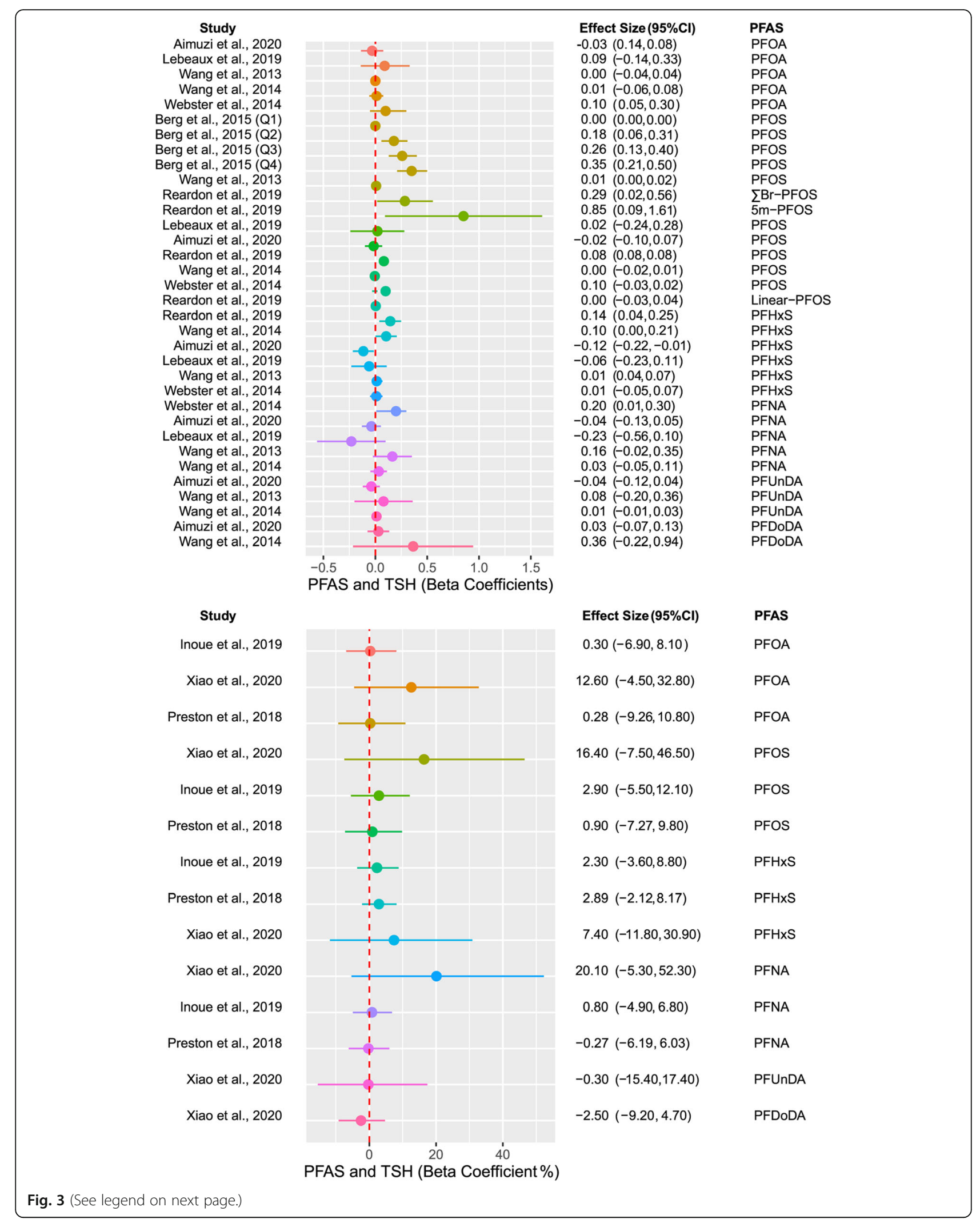


(See figure on previous page.)

Fig. 3 Summary statistics of selected PFAS on thyrotropin (TSH) in the epidemiologic literature. Displayed is a representative forest plot of the PFAS and TSH relationship for selected PFAS where not all units are the same across all studies. The aim is to assess the overall direction and strength of association. Abbreviations: PFOA, perfluorooctanoic acid; PFOS, perfluorooctane sulfonate; PFHxS, perfluorohexane sulfonic acid; PFNA, perfluorononanoic acid; PFUnDA, perfluoroundecanoate; PFDoDA, perfluorododecanoate; TSH, thyrotropin

review examined the impact of PFAS on fetal THs by measuring the association of PFAS levels with cord blood and neonatal heel stick TH levels [80, 81, 83, 86, 87]. While results varied, maternal PFAS levels were generally positively associated with fetal and neonatal $\mathrm{TSH}$ and negatively with $\mathrm{T} 3$ and $\mathrm{T} 4$. THs regulate metabolic and physiological pathways that determine pregnancy outcomes (i.e. birth size) and development (i.e. neurodevelopment) [35]. Therefore, in addition to affecting maternal health, PFAS thyrotoxicity in pregnancy may alter fetal growth and development, with longlasting impacts on child health.

\section{Future directions: epidemiological modeling of PFAS and gestational diabetes mellitus (GDM)}

To accurately estimate the association of PFAS and GDM risk, study design plays an important role. Longitudinal, unlike cross-sectional, studies allow for the identification of temporality in the relationship. For example, Reardon et al. found that PFHxS was positively associated with TSH and negatively with FT4 [82]. The association was time-dependent for TSH, with the effect size decreasing as gestation progressed [82]. Measuring biomarkers at standardized points throughout gestation can yield less biased conclusions on the relationships proposed here and improve generalizability and transportability to other populations.

This pathway approach primarily strengthens the potential for causal inference by way of a more accurate and precise measurement of the phenomenon of interest. It reduces bias due to measurement error by capturing information on physiologically relevant processes and by including multiple measures within an individual. If biomarkers are measured longitudinally in pregnancy, that likewise strengthens the potential for measuring a causal effect.

The subdiscipline in epidemiology called 'causal inference' lays out specific techniques for estimation, inference, and quantitative analysis of bias [90, 91]. The basis of this approach is the potential outcomes framework. This is a hypothetical framework by which all people who are unexposed in a study and have a set of outcomes can essentially be taken back in time, and then be given the exposure in order to measure their set of outcomes under that condition. The probability of outcome under the two scenarios can be compared to get a causal effect estimate. This is not feasible in real life. The objective is to approximate this scenario by applying analytical techniques. Most pregnant women are exposed to PFAS so the idea of a no exposure group is also unrealistic. For this reason, we might instead estimate the difference in risk if all pregnant women had PFAS levels at the 75th percentile versus all women who had levels at the 25th percentile.

A first step in this approach is to generate a directed acyclic graph (DAG) that can structure the relationship of the exposure and the outcome in relationship to confounders, intermediate variables, and colliders [92]. This is a way to identify sources of structural bias that can be minimized by collecting information, adjustment, or inverse probability weighting. Confounders are those variables which are common causes of the $\mathrm{X}$ (exposure), the $M$ (intermediate), and $Y$ (outcome) variables in three categories: 1) confounders of PFAS (X) and GDM (Y); 2) confounders of PFAS $(\mathrm{X})$ and intermediate biomarkers ( $\mathrm{M}$, maternal thyroid and pancreas, fetal placenta); and 3) confounders of intermediate biomarkers (M) and GDM (Y). Effect modifiers are variables such as thyroid autoimmune biomarkers which might be operating in tandem with PFAS exposure in some fashion but may not necessarily be intermediates in a causal pathway. Effect modifiers can be identified and evaluated based on knowledge of mechanism and previously reported findings, but they cannot be represented in a DAG [93]. Analytical strategies for constructing DAGs are described elsewhere [92, 94, 95]. Statistical approaches to analyze these associations are outside of the scope of this review, but also an important area in developing this pathway-level approach. G methods have been proposed for the analysis of complex, longitudinal data with timevarying confounders [96]. Methods that allow for single or multiple mediators may also be useful in estimation of PFAS effects in the presence of measures of placental, thyroid, liver, and/or pancreatic function [97].

Adjustment for variables that are not confounders can also lead to bias in the estimation of the exposure and outcome association [98]. Variables that were adjusted for but may not be confounders in the studies reviewed are noted in Supplemental Table 1. To qualify as a confounder, the variable must precede the exposure in time and plausibly be a common cause of the exposure and the outcome.

\section{Discussion}

The evidence of PFAS exposure as a direct cause of GDM in the epidemiological literature is weak. This 
conclusion could change in future studies that consider the indirect pathway and measure the biomarkers proposed here. Based on the results of this review, we theorize that PFAS can alter TH homeostasis in pregnancy by causing higher TSH levels and lower T3 and T4 levels, disrupting downstream glucose metabolism. $\mathrm{TH}$ disruption may be happening at the level of the thyroid gland, the hypothalamus, the pituitary, or the fetal placenta (Fig. 1).

There is experimental evidence of the toxicity of PFAS to the thyroid. PFOS and PFHxS were found to inhibit iodide uptake in the FRTL-5 rat thyroid follicular cell line $[99,100]$. These effects were not seen with PFOA, indicating compound specific effects on the thyroid [99]. In zebrafish, PFUnDA and PFOA induced changes in the expression of genes involved in $\mathrm{TH}$ metabolism and excretion [101, 102] while perfluorotridecanoic acid (PFTrDA) upregulated genes related to $\mathrm{TH}$ activation and synthesis [101]. Furthermore, PFAS may competitively bind to $\mathrm{TH}$ binding proteins to displace T4 [103] and to increase transcript expression of D1, an enzyme that converts T4 to T3, in the thyroids of rats [104]. All together, PFAS may therefore be altering $\mathrm{TH}$ homeostasis by direct interaction with the thyroid gland and through mechanisms that control TH availability. Based on the results of our literature review, PFAS may also exacerbate underlying thyroid autoimmunity. Approximately $30 \%$ of pregnant women during early pregnancy were found to be TBOAb/TgAb positive [105], confirming that this is a variable that cannot be ignored.

From our findings, we infer that PFAS exposure also causes glucose and insulin levels to increase in pregnancy. We propose that changes in glucose and insulin levels here may be a secondary consequence of thyroid dysregulation. This may also reflect direct toxicity of PFAS to the maternal liver and/or pancreas that could disrupt maternal and fetal glucose homeostasis during pregnancy. Molecular docking analysis found that PFOS, PFOA, and PFHxS can directly bind multiple peroxisome proliferator-activated receptor (PPAR) isoforms in the liver that regulate glucose metabolism [106]. Glycogen depletion and mitochondrial dysfunction are PPARindependent mechanisms by which PFAS exerts toxicity in the liver [107]. In carp, a closely related species to zebrafish, PFOS exposure inhibited liver glucokinase gene expression, which regulates glucose uptake into the liver, and depleted glycogen stores in the liver, therefore altering glucose homeostasis [108]. Glycogen depletion was also observed in the livers of pregnant mice exposed to PFAS, along with hepatocellular alterations, such as hypertrophy and necrosis [109]. Additionally, Sant et al. determined that zebrafish embryonic exposure to PFAS induced morphological changes to the pancreas of zebrafish larvae, including reduced $\beta$ cell area for insulin production, as well as reduced gene expression of hormones related to glucoregulation [110].

The majority of PFAS examined demonstrated null associations with GDM and the biomarkers proposed in this causal pathway. This may be a result of limitations in study design when examining PFAS cross-sectionally or sampling cases and controls versus a prospective cohort design. Findings could also be null due to measurement error or incorrect model specification. Incorrect model specification here includes the omission of interactions or intermediate variables. These are all issues that the proposed pathway approach can address. Another issue is that due to this being a large and diverse class of chemicals, the toxicological profiles of different PFAS vary dramatically. Therefore, there may be only particular compounds that impact the specific biomarkers proposed in this pathway. Biomarker-based studies are needed to narrow these lists and identify these specific relationships. Additionally, human exposure to PFAS is typically through complex mixtures and multiple routes of exposure, rather than to individual compounds [1]. In mixtures, the compounds have been shown to exert both additive and synergistic toxicities; therefore, examining the overall mixture effect may be more accurate in depicting the human exposure [111]. Few studies included in our review examined the combined effects of the PFAS on the outcomes. Chan et al. examined the association of the molar sum of PFHxS, PFOA, and PFOS with hypothyroxinemia in pregnant women but found no additive or synergistic effect, as compared to analyzing each compound alone [77]. Three studies [58, 70, 80] used Bayesian Kernel Machine Regression (BKMR) as a statistical approach to determine the PFAS mixture effect while also providing associations for each compound in the mixture to understand which are the main drivers of the overall association [112]. Correlations between mixture components are also taken into account [112]. With this approach, a PFAS mixture (six compounds) was associated with plasma glucose levels [58]. The single compounds PFOS and 2-(N-methyl-perfluorooctane sulfonamide) acetate (N-MeFOSAA) were the main contributors to this overall association [58]. Mixture modeling can be useful in identifying the 'bad actors.' There is no perfect model to account for these complex relationships, which is one reason it is impossible to conclude that PFAS associations with GDM are truly null.

Impairments in placental function can affect maternal health during pregnancy (i.e. preeclampsia). It follows that PFAS-induced effects on the placenta may also adversely affect the mother, as well as the fetus. The placenta is exposed to PFAS from maternal circulation [113]. In animal models, PFAS exposure causes both increases and decreases in placental weight, inhibits 
activity and gene expression of placental hormones, and induces necrosis and histopathological alterations, with compound specific effects $[109,114,115]$. Rodents do not produce hCG, so this model does not translate directly. Placental biomarkers altered by PFAS exposure during pregnancy, including but not exclusive to hCG, should be prioritized when studying this causal pathway.

Reverse causation, whereby thyroid dysfunction precedes and causes physiological differences that affect maternal PFAS concentrations, cannot be eliminated. For example, THs regulate multiple processes in the kidney, including the glomerular filtration rate (GFR), which is the rate of fluid filtered by the kidneys. Renal clearance is the primary mode of excretion of PFAS and a lower GFR is associated with higher serum PFAS levels [116]. Any alteration in thyroid function could in theory change the GFR and rate of excretion of PFAS, therefore affecting serum levels of PFAS. Watkins et al. measured the direction of association between PFOA and kidney function by comparing measured versus modelpredicted (based on environmental exposure estimates) serum PFOA levels, which are dependently and independently influenced by GFR, respectively [116]. Only the measured PFOA was associated with kidney dysfunction, which may indicate that PFAS is a result of rather than the cause of the kidney function. Other physiological processes have been proposed that may demonstrate reverse causality in the causal association of PFAS with health outcomes [117]. Adding confounders related to circulating PFAS levels, such as GFR, into the model of PFAS exposure and GDM development could address this issue of reverse causation, but it cannot be completely eliminated when measures are cross-sectional in pregnancy.

The pathway proposed here may be influenced by a broad range of environmental exposures beyond PFAS. For example, organochlorine pesticides (OCPs) used for crop protection, such as dichlorodiphenyl trichloroethane and hexachlorobenzene, are also potential candidates for disruption of thyroid function [118, 119]. Several OCPs were associated with TSH and FT4 levels in cord plasma [120] and with the risk of developing GDM [121]. Likewise, exposure to brominated flame retardants, such as polybrominated diphenyl ethers (PBDEs), have been positively associated with GDM risk [122]. PBDEs are negatively associated with total T3 and T4 in cord serum and with altered birth outcomes, including lower IQ [123-125]. Furthermore, endocrine disruptors in plastics and personal care items, including phthalates and bisphenol $\mathrm{A}$, have been shown to affect $\mathrm{TH}$ regulation including impairment of iodine uptake and inhibition of $\mathrm{TH}$ homeostasis [126] and phthalates have been found to be associated with hCG, glucose intolerance, and GDM [127-129].
GDM is a common pregnancy complication that may progress to adverse maternal and child health outcomes and can be better appreciated as a consequence of environmental exposure preconception and during pregnancy. Here, we present the evidence to date on PFAS as a relevant exposure. On the clinical side, this knowledge can be used to distinguish patients at different levels of risk for GDM and conversion of GDM to T2D, and also to formulate stronger individual-level interventions related to PFAS exposures through diet and drinking water in pregnancy. On the level of public health, these types of studies can be useful in generating causal knowledge on population-level exposures that increase the risk of GDM to motivate interventions when supported by the evidence. If consensus is reached regarding specific and harmful effects of PFAS, these types of studies could motivate innovation in the design of chemicals used in food packaging and commercial products. The biomarkers proposed in this pathway can be monitored in large cohorts to evaluate efficacy of PFAS regulation and/or more targeted types of interventions.

\section{Conclusions}

Epidemiological evidence strongly supports that problems with thyroid function can disrupt glucose homeostasis, which we posit may be a driving force for the development of GDM and may be a mechanism through which PFAS exposures exert toxicity in pregnant women. This mechanism may offer an opportunity to more precisely and accurately quantify the associations of PFAS (and other thyroid-disrupting chemicals) with GDM. The relationship between $\mathrm{TH}$ biomarkers and GDM as well as glucose biomarkers and GDM are well established, however the combination of these two associations into one common pathway is relatively unexplored. PFAS exposure may be an upstream environmental lever that interferes with thyroid function and disrupts downstream glucose homeostasis.

\section{Abbreviations \\ AITDs: Autoimmune thyroid diseases; D1: Type 1 iodothyronine deiodinases; D2: Type 2 iodothyronine deiodinases; D3: Type 3 iodothyronine deiodinases; FFA: Free fatty acid; FT3: Free triiodothyronine; FT4: Free thyroxine; GDM: Gestational diabetes mellitus; hCG: Human chorionic gonadotropin; HOMA: Homeostasis model assessment; PLGF: Placental growth factor; PFAS: Per- and polyfluoroalkyl substances; T1D: Type I diabetes; T2D: Type II diabetes; T3: Triiodothyronine; T4: Thyroxine; TBG: Thyroxine-binding globulin; Tg: Thyroglobulin; TgAb: Thyroglobulin antibody; TH: Thyroid hormone; TPO: Thyroidperoxidase; TPOAb: Thyroid peroxidase antibody; $\mathrm{TRH}$ : Thyrotropin-releasing hormone; TSH: Thyrotropin}

\section{Supplementary Information}

The online version contains supplementary material available at https://doi. org/10.1186/s12940-021-00740-z.

Additional file 1: Methods. Supplemental Table 1. Summary of studies examining PFAS and gestational diabetes (GDM), glucose, or 
thyroid biomarkers in pregnancy. Supplemental Table 2. Summary of PFAS and biomarker levels in cohorts. Supplemental Table $\mathbf{3}$.

Association of PFAS with gestational diabetes mellitus (GDM).

Supplemental Table 4. Association of PFAS with glucose and insulin biomarkers. Supplemental Table 5: Evidence of PFAS association with free and total triiodothyronine (T3) in pregnancy. Supplemental Table 6: Evidence of PFAS association with free and total thyroxine (T4) in pregnancy. Supplemental Table 7: Evidence of PFAS association with thyrotropin (TSH) in pregnancy. Supplemental Table 8: Evidence of PFAS association with thyroid hormones by thyroid autoantibody status in pregnancy.

\section{Acknowledgements}

This literature review was initiated in response to the National Institute of Environmental Health Sciences Funding initiative entitled, 'Pregnancy as a Vulnerable Time Period for Women's Health (RFA-ES-20-003)'. We acknowledge the support of Dr. Esa Davis in the initiation of this project. Figure 1 was created with BioRender.com.

\section{Authors' contributions}

JA conceptualized the study and oversaw the analysis. RLB, HW, FF, MB conducted the literature review, completed the analysis, and drafted the manuscript. RLB and JJA led the writing and editing of the manuscript. MF, CAN, CLH, DDM, and JC provided critical feedback. All authors read and approved the final manuscript

\section{Funding}

This work was supported by 5R01ES029336-02, National Institutes of Health (J.J.A., R.L.B., H.L) and 1R56Al139189-01, National Institutes of Health (F.F. C.L.H.) and the Department of Epidemiology, University of Pittsburgh Graduate School of Public Health.

\section{Availability of data and materials}

All data generated or analyzed during this study are included in this published article [and its supplementary information files].

\section{Declarations}

Ethics approval and consent to participate

Not applicable.

\section{Consent for publication}

Not applicable.

\section{Competing interests}

The authors declare that they have no competing interests.

\section{Author details}

'Department of Epidemiology, Graduate School of Public Health, University of Pittsburgh, Pittsburgh, PA, USA. 'Department of Civil and Environmental Engineering, University of Pittsburgh, Pittsburgh, PA, USA. ${ }^{3}$ Department of Obstetrics, Gynecology, and Reproductive Sciences, University of Pittsburgh School of Medicine, Pittsburgh, PA, USA. " Environmental and Occupational Health, Graduate School of Public Health, University of Pittsburgh, Pittsburgh, PA, USA.

Received: 28 December 2020 Accepted: 27 April 2021

Published online: 22 May 2021

\section{References}

1. Sunderland EM, Hu XC, Dassuncao C, Tokranov AK, Wagner CC, Allen JG. A review of the pathways of human exposure to poly- and perfluoroalkyl substances (PFASs) and present understanding of health effects. J Expo Sci Environ Epidemiol. 2019;29(2):131-47. https://doi.org/10.1038/s41370-0180094-1.

2. Faithfull NS, Weers JG. Perfluorocarbon compounds. Vox Sang. 1998; 74(Suppl 2):243-8. https://doi.org/10.1111/j.1423-0410.1998.tb05426.x.

3. Kato K, Wong LY, Jia LT, Kuklenyik Z, Calafat AM. Trends in exposure to polyfluoroalkyl chemicals in the U.S. population: 1999-2008. Environ Sci Technol. 2011;45(19):8037-45.
4. Bjerregaard-Olesen C, Bossi R, Liew Z, Long M, Bech BH, Olsen J, Henriksen TB, Berg V, Nøst TH, Zhang JJJljoh, et al. Maternal serum concentrations of perfluoroalkyl acids in five international birth cohorts. Int J Hyg Environ Health. 2017;220(2):86-93.

5. $\mathrm{Ng}$ CA, Hungerbühler K. Bioaccumulation of perfluorinated alkyl acids: observations and models. Environ Sci Technol. 2014;48(9):4637-48. https:// doi.org/10.1021/es404008g

6. Li Y, Fletcher T, Mucs D, Scott K, Lindh CH, Tallving P, et al. Half-lives of PFOS, PFHXS and PFOA after end of exposure to contaminated drinking water. Occup Environ Med. 2018;75(1):46-51. https://doi.org/10.1136/ oemed-2017-104651.

7. Xu Y, Fletcher T, Pineda D, Lindh CH, Nilsson C, Glynn A, et al. Serum halflives for short- and Long-chain Perfluoroalkyl acids after ceasing exposure from drinking water contaminated by firefighting foam. Environ Health Perspect. 2020;128(7):77004. https://doi.org/10.1289/EHP6785.

8. Tian Y, Zhou Y, Miao M, Wang Z, Yuan W, Liu X, et al. Determinants of plasma concentrations of perfluoroalkyl and polyfluoroalkyl substances in pregnant women from a birth cohort in Shanghai, China. Environ Int. 2018; 119:165-73. https://doi.org/10.1016/j.envint.2018.06.015.

9. Plows JF, Stanley JL, Baker PN, Reynolds CM, Vickers MH. The Pathophysiology of Gestational Diabetes Mellitus. Int J Mol Sci. 2018;19(11): 3342

10. Ogurtsova K, da Rocha Fernandes JD, Huang Y, Linnenkamp U, Guariguata L, Cho NH, et al. IDF Diabetes atlas: global estimates for the prevalence of diabetes for 2015 and 2040. Diabetes Res Clin Pract. 2017;128:40-50. https:// doi.org/10.1016/j.diabres.2017.03.024.

11. Parsons J, Sparrow K, Ismail K, Hunt K, Rogers H, Forbes A. Experiences of gestational diabetes and gestational diabetes care: a focus group and interview study. BMC Pregnancy Childbirth. 2018;18(1):25. https://doi.org/1 0.1186/s12884-018-1657-9.

12. Mullur R, Liu YY, Brent GA. Thyroid hormone regulation of metabolism. Physiol Rev. 2014;94(2):355-82. https://doi.org/10.1152/physrev.00030.2013.

13. Johnson J. Diabetes control in thyroid disease. Diabetes Spectrum. 2006; 19(3):148-53. https://doi.org/10.2337/diaspect.19.3.148.

14. Brent GA. Mechanisms of thyroid hormone action. J Clin Invest. 2012;122(9): 3035-43. https://doi.org/10.1172/JCl60047.

15. Cicatiello AG, Di Girolamo D, Dentice M. Metabolic effects of the intracellular regulation of thyroid hormone: old players, new concepts. Front Endocrinol (Lausanne). 2018;9:474. https://doi.org/10.3389/fendo.2018.00474.

16. Park EA, Song S, Vinson C, Roesler WJ. Role of CCAAT enhancer-binding protein beta in the thyroid hormone and CAMP induction of phosphoenolpyruvate carboxykinase gene transcription. J Biol Chem. 1999; 274(1):211-7. https://doi.org/10.1074/jbc.274.1.211.

17. Brenta G. Why can insulin resistance be a natural consequence of thyroid dysfunction? J Thyroid Res. 2011;2011:152850

18. Hage M, Zantout MS, Azar ST. Thyroid disorders and diabetes mellitus. J Thyroid Res. 2011:2011:439463.

19. Walsh JP. Managing thyroid disease in general practice. Med J Aust. 2016; 205(4):179-84. https://doi.org/10.5694/mja16.00545.

20. Taylor PN, Albrecht D, Scholz A, Gutierrez-Buey G, Lazarus JH, Dayan CM, et al. Global epidemiology of hyperthyroidism and hypothyroidism. Nat Rev Endocrinol. 2018;14(5):301-16. https://doi.org/10.1038/nrendo.2018.18.

21. Hwang GB, Yoon JS, Park KJ, Lee HS, Hwang JS. Prevalence of autoimmune thyroiditis in patients with type 1 diabetes: a long-term follow-up study. Ann Pediatr Endocrinol Metab. 2018;23(1):33-7. https://doi.org/10.6065/a pem.2018.23.1.33

22. Merrill SJ, Mu Y. Thyroid autoimmunity as a window to autoimmunity: an explanation for sex differences in the prevalence of thyroid autoimmunity. J Theor Biol. 2015;375:95-100. https://doi.org/10.1016/j.jtbi.2014.12.015.

23. Brent GA. Environmental exposures and autoimmune thyroid disease. Thyroid. 2010;20(7):755-61. https://doi.org/10.1089/thy.2010.1636.

24. Tal R, Taylor HS, Burney RO, Mooney SB, Giudice LC. Endocrinology of Pregnancy. In: Endotext Edited by Feingold KR, Anawalt B, Boyce A, Chrousos G, Dungan K, Grossman A, Hershman JM, Kaltsas G, Koch C, Kopp P et al. South Dartmouth; MDText.com, Inc;; 2000. Available from: https:// www.ncbi.nIm.nih.gov/books/NBK278962/.

25. Glinoer $D$. The regulation of thyroid function in pregnancy: pathways of endocrine adaptation from physiology to pathology. Endocr Rev. 1997;18(3): 404-33. https://doi.org/10.1210/edrv.18.3.0300.

26. Korevaar Tl, de Rijke YB, Chaker L, Medici M, Jaddoe WW, Steegers EA et al. Stimulation of thyroid function by human chorionic gonadotropin 
during pregnancy: a risk factor for thyroid disease and a mechanism for known risk factors. Thyroid. 2017;27(3):440-50. https://doi.org/10.1089/ thy.2016.0527.

27. Korevaar TI, Steegers EA, de Rijke YB, Visser WE, Jaddoe WW, Visser TJ, et al. Placental Angiogenic factors are associated with maternal thyroid function and modify hCG-mediated FT4 stimulation. J Clin Endocrinol Metab. 2015; 100(10):E1328-34. https://doi.org/10.1210/jc.2015-2553.

28. Korevaar TIM, Medici M, Visser TJ, Peeters RP. Thyroid disease in pregnancy: new insights in diagnosis and clinical management. Nat Rev Endocrinol. 2017:13(10):610-22. https://doi.org/10.1038/nrendo.2017.93.

29. Vissenberg R, Manders VD, Mastenbroek S, Fliers E, Afink GB, Ris-Stalpers C, et al. Pathophysiological aspects of thyroid hormone disorders/thyroid peroxidase autoantibodies and reproduction. Hum Reprod Update. 2015; 21(3):378-87. https://doi.org/10.1093/humupd/dmv004

30. Moog NK, Entringer S, Heim C, Wadhwa PD, Kathmann N, Buss C. Influence of maternal thyroid hormones during gestation on fetal brain development. Neuroscience. 2017;342:68-100. https://doi.org/10.1016/j.neuroscience.2015. 09.070 .

31. James SR, Franklyn JA, Kilby MD. Placental transport of thyroid hormone Best Pract Res Clin Endocrinol Metab. 2007;21(2):253-64. https://doi.org/10.1 016/j.beem.2007.03.001.

32. Chan SY, Vasilopoulou E, Kilby MD. The role of the placenta in thyroid hormone delivery to the fetus. Nat Clin Pract Endocrinol Metab. 2009;5(1): 45-54. https://doi.org/10.1038/ncpendmet1026.

33. Maraka S, Ospina NM, O'Keeffe DT, Espinosa De Ycaza AE, Gionfriddo MR, Erwin PJ, et al. Subclinical hypothyroidism in pregnancy: a systematic review and meta-analysis. Thyroid. 2016;26(4):580-90. https://doi.org/10.1089/thy.2 015.0418 .

34. Morreale de Escobar G, Obregon MJ, Escobar del Rey F. Role of thyroid hormone during early brain development. Eur J Endocrinol. 2004;151(Suppl 3):U25-37.

35. Forhead AJ, Fowden AL. Thyroid hormones in fetal growth and prepartum maturation. J Endocrinol. 2014;221(3):R87-r103. https:/doi.org/10.1530/JOE-14-0025.

36. Liu Y, Guo F, Maraka S, Zhang Y, Zhang C, Korevaar T, et al. Associations between human chorionic gonadotropin, maternal free Thyroxine, and gestational Diabetes mellitus. Thyroid. 2021. https://doi.org/10.1089/thy.202 0.0920 .

37. Donovan BM, Nidey NL, Jasper EA, Robinson JG, Bao W, Saftlas AF, et al. First trimester prenatal screening biomarkers and gestational diabetes mellitus: a systematic review and meta-analysis. Plos One. 2018;13(7): e0201319. https://doi.org/10.1371/journal.pone.0201319.

38. American Diabetes A. Diagnosis and classification of diabetes mellitus. Diabetes Care. 2010;33(Suppl 1):S62-9. https://doi.org/10.2337/dc10-S062.

39. Leng J, Li W, Wang L, Zhang S, Liu H, Li W, et al. Higher thyroid-stimulating hormone levels in the first trimester are associated with gestational diabetes in a Chinese population. Diabet Med. 2019;36(12):1679-85. https:// doi.org/10.1111/dme.14106

40. Rawal S, Tsai MY, Hinkle SN, Zhu Y, Bao W, Lin Y, et al. A longitudinal study of thyroid markers across pregnancy and the risk of gestational Diabetes. J Clin Endocrinol Metab. 2018;103(7):2447-56. https://doi.org/10.1210/jc.201702442.

41. Gong LL, Liu H, Liu LH. Relationship between hypothyroidism and the incidence of gestational diabetes: a meta-analysis. Taiwan J Obstet Gynecol. 2016;55(2):171-5. https://doi.org/10.1016/j.tjog.2016.02.004.

42. Aggarawal N, Suri V, Singla R, Chopra S, Sikka P, Shah VN, et al. Pregnancy outcome in hyperthyroidism: a case control study. Gynecol Obstet Investig 2014;77(2):94-9. https://doi.org/10.1159/000357615

43. Yang Y, Li Q, Wang Q, Ma X. Thyroid antibodies and gestational diabetes mellitus: a meta-analysis. Fertil Steril. 2015;104(3):665-71 e663. https://doi. org/10.1016/j.fertnstert.2015.06.003.

44. Jia M, Wu Y, Lin B, Shi Y, Zhang Q, Lin Y, et al. Meta-analysis of the association between maternal subclinical hypothyroidism and gestational diabetes mellitus. Int J Gynaecol Obstet. 2019;144(3):239-47. https://doi. org/10.1002/ijgo.12751

45. Huang $K, X u Y$, Yan $S$, Li T, Xu Y, Zhu P, et al. Isolated effect of maternal thyroid-stimulating hormone, free thyroxine and antithyroid peroxidase antibodies in early pregnancy on gestational diabetes mellitus: a birth cohort study in China. Endocr J. 2019;66(3):223-31. https://doi.org/10.1507/ endocrj.EJ18-0340.

46. Wallace TM, Levy JC, Matthews DR. Use and abuse of HOMA modeling. Diabetes Care. 2004;27(6):1487-95. https://doi.org/10.2337/ diacare.27.6.1487
47. American Diabetes A. 14. Management of Diabetes in pregnancy: standards of medical Care in Diabetes-2020. Diabetes Care. 2020;43(Suppl 1):S183-92. https://doi.org/10.2337/dc20-S014.

48. Matthews DR, Hosker JP, Rudenski AS, Naylor BA, Treacher DF, Turner RC. Homeostasis model assessment: insulin resistance and beta-cell function from fasting plasma glucose and insulin concentrations in man. Diabetologia. 1985;28(7):412-9. https://doi.org/10.1007/BF00280883.

49. Matsuda M, DeFronzo RA. Insulin sensitivity indices obtained from oral glucose tolerance testing: comparison with the euglycemic insulin clamp. Diabetes Care. 1999:22(9):1462-70. https://doi.org/10.2337/diacare.22.9.1462.

50. Leighton E, Sainsbury CA, Jones GC. A practical review of C-peptide testing in Diabetes. Diab Ther. 2017;8(3):475-87. https://doi.org/10.1007/s13300-0170265-4.

51. Sheehan MT. Biochemical Testing of the Thyroid: TSH is the Best and, Oftentimes, Only Test Needed - A Review for Primary Care. Clin Med Res. 2016;14(2):83-92. https://doi.org/10.3121/cmr.2016.1309.

52. McNeil AR, Stanford PE. Reporting thyroid function tests in pregnancy. Clin Biochem Rev. 2015;36(4):109-26.

53. Spencer CA. Assay of thyroid hormones and related substances. In: Feingold KR, Anawalt B, Boyce A, Chrousos G, Dungan K, Grossman A, Hershman JM, Kaltsas G, Koch C, Kopp P, et al., editors. Endotext. South Dartmouth: MDText.com, Inc.; 2000.

54. Welsh KJ, Soldin SJ. DIAGNOSIS OF ENDOCRINE DISEASE: how reliable are free thyroid and total T3 hormone assays? Eur J Endocrinol. 2016;175(6): R255-63. https://doi.org/10.1530/EJE-16-0193.

55. Soldin OP, Soldin SJ. Thyroid hormone testing by tandem mass spectrometry. Clin Biochem. 2011;44(1):89-94. https://doi.org/10.1016/j. clinbiochem.2010.07.020.

56. Liu X, Zhang L, Chen L, Li J, Wang Y, Wang J, et al. Structure-based investigation on the association between perfluoroalkyl acids exposure and both gestational diabetes mellitus and glucose homeostasis in pregnant women. Environ Int. 2019;127:85-93. https://doi.org/10.1016/j.envint.2019.03.035.

57. Matilla-Santander N, Valvi D, Lopez-Espinosa MJ, Manzano-Salgado CB, Ballester F, Ibarluzea J, et al. Exposure to Perfluoroalkyl substances and metabolic outcomes in pregnant women: evidence from the Spanish INMA birth cohorts. Environ Health Perspect. 2017;125(11):117004. https://doi.org/1 0.1289/EHP1062.

58. Preston EV, Rifas-Shiman SL, Hivert MF, Zota AR, Sagiv SK, Calafat AM, Oken E, James-Todd T. Associations of Per- and Polyfluoroalkyl Substances (PFAS) With Glucose Tolerance During Pregnancy in Project Viva. J Clin Endocrinol Metab. 2020;105(8):e2864-e2876.

59. Rahman ML, Zhang C, Smarr MM, Lee S, Honda M, Kannan K, et al. Persistent organic pollutants and gestational diabetes: a multi-center prospective cohort study of healthy US women. Environ Int. 2019;124:24958. https://doi.org/10.1016/j.envint.2019.01.027.

60. Shapiro GD, Dodds L, Arbuckle TE, Ashley-Martin J, Ettinger AS, Fisher M, et al. Exposure to organophosphorus and organochlorine pesticides, perfluoroalkyl substances, and polychlorinated biphenyls in pregnancy and the association with impaired glucose tolerance and gestational diabetes mellitus: the MIREC study. Environ Res. 2016;147:71-81. https://doi.org/10.1 016/j.envres.2016.01.040

61. Wang H, Yang J, Du H, Xu L, Liu S, Yi J, et al. Perfluoroalkyl substances, glucose homeostasis, and gestational diabetes mellitus in Chinese pregnant women: a repeat measurement-based prospective study. Environ Int. 2018; 114:12-20. https://doi.org/10.1016/j.envint.2018.01.027.

62. Zhang C, Sundaram R, Maisog J, Calafat AM, Barr DB, Buck Louis GM. A prospective study of prepregnancy serum concentrations of perfluorochemicals and the risk of gestational diabetes. Fertil Steril. 2015; 103(1):184-9. https://doi.org/10.1016/j.fertnstert.2014.10.001.

63. Xu H, Zhou Q, Zhang J, Chen X, Zhao H, Lu H, et al. Exposure to elevated perand polyfluoroalkyl substances in early pregnancy is related to increased risk of gestational diabetes mellitus: a nested case-control study in Shanghai, China. Environ Int. 2020;143:105952. https://doi.org/10.1016/j.envint.2020.105952.

64. Wang $Y$, Zhang L, Teng Y, Zhang J, Yang L, Li J, et al. Association of serum levels of perfluoroalkyl substances with gestational diabetes mellitus and postpartum blood glucose. J Environ Sci (China). 2018;69:5-11. https://doi. org/10.1016/j.jes.2018.03.016.

65. Li J, Yao J, Xia W, Dai J, Liu H, Pan Y, et al. Association between exposure to per- and polyfluoroalkyl substances and blood glucose in pregnant women. Int J Hyg Environ Health. 2020;230:113596. https://doi.org/10.1016/j.jiheh.202 0.113596 
66. Valvi D, Oulhote $Y$, Weihe P, Dalgård C, Bjerve KS, Steuerwald U, et al. Gestational diabetes and offspring birth size at elevated environmental pollutant exposures. Environ Int. 2017;107:205-15. https://doi.org/10.1016/j. envint.2017.07.016.

67. Jensen RC, Glintborg D, Timmermann CAG, Nielsen F, Kyhl HB, Andersen $H R$, et al. Perfluoroalkyl substances and glycemic status in pregnant Danish women: the Odense child cohort. Environ Int. 2018;116:101-7. https://doi. org/10.1016/j.envint.2018.04.010.

68. Starling AP, Adgate $J$, Hamman RF, Kechris K, Calafat AM, Ye X, et al. Perfluoroalkyl substances during pregnancy and offspring weight and adiposity at birth: examining mediation by maternal fasting glucose in the healthy start study. Environ Health Perspect. 2017;125(6):067016. https://doi. org/10.1289/EHP641.

69. Ren $Y$, Jin L, Yang F, Liang H, Zhang $Z$, Du J, et al. Concentrations of perfluoroalkyl and polyfluoroalkyl substances and blood glucose in pregnant women. Environ Health. 2020;19(1):88. https://doi.org/10.1186/s12 940-020-00640-8.

70. Mehta SS, James-Todd T, Applebaum KM, Bellavia A, Coleman-Phox K, Adler $\mathrm{N}$, et al. Persistent organic pollutants and maternal glycemic outcomes in a diverse pregnancy cohort of overweight women. Environ Res. 2021;193: 110551. https://doi.org/10.1016/j.envres.2020.110551.

71. Cardenas A, Gold DR, Hauser R, Kleinman KP, Hivert MF, Calafat AM, et al. Plasma concentrations of per- and Polyfluoroalkyl substances at baseline and associations with glycemic indicators and Diabetes incidence among high-risk adults in the Diabetes prevention program trial. Environ Health Perspect. 2017;125(10):107001. https://doi.org/10.1289/EHP1612.

72. Duan Y, Sun H, Yao Y, Meng Y, Li Y. Distribution of novel and legacy per -/polyfluoroalkyl substances in serum and its associations with two glycemic biomarkers among Chinese adult men and women with normal blood glucose levels. Environ Int. 2020;134:105295. https://doi.org/10.1016/j. envint.2019.105295.

73. Melzer D, Rice N, Depledge MH, Henley WE, Galloway TS. Association between serum perfluorooctanoic acid (PFOA) and thyroid disease in the U. S. National Health and nutrition examination survey. Environ Health Perspect. 2010;118(5):686-92. https://doi.org/10.1289/ehp.0901584.

74. Kim MJ, Moon S, Oh BC, Jung D, Ji K, Choi K, et al. Association between perfluoroalkyl substances exposure and thyroid function in adults: a metaanalysis. Plos One. 2018;13(5):e0197244. https://doi.org/10.1371/journal.pone. 0197244.

75. Aimuzi R, Luo K, Huang R, Huo X, Nian M, Ouyang F, et al. Perfluoroalkyl and polyfluroalkyl substances and maternal thyroid hormones in early pregnancy. Environ Pollut. 2020;264:114557.

76. Berg V, Nøst TH, Hansen S, Elverland A, Veyhe AS, Jorde R, et al. Assessing the relationship between perfluoroalkyl substances, thyroid hormones and binding proteins in pregnant women; a longitudinal mixed effects approach. Environ Int. 2015;77:63-9. https://doi.org/10.1016/j.envint.2015.01.007.

77. Chan E, Burstyn I, Cherry N, Bamforth F, Martin JW. Perfluorinated acids and hypothyroxinemia in pregnant women. Environ Res. 2011;111(4):559-64. https://doi.org/10.1016/j.envres.2011.01.011.

78. Inoue K, Ritz B, Andersen SL, Ramlau-Hansen CH, Hoyer BB, Bech BH, et al. Perfluoroalkyl substances and maternal thyroid hormones in early pregnancy; findings in the Danish national birth cohort. Environ Health Perspect. 2019;127(11):117002. https://doi.org/10.1289/EHP5482.

79. Itoh S, Araki A, Miyashita C, Yamazaki K, Goudarzi H, Minatoya M, et al. Association between perfluoroalkyl substance exposure and thyroid hormone/thyroid antibody levels in maternal and cord blood: The Hokkaido Study. Environ Int. 2019;133(Pt A):105139.

80. Lebeaux RM, Doherty BT, Gallagher LG, Zoeller RT, Hoofnagle AN, Calafat $A M$, et al. Maternal serum perfluoroalkyl substance mixtures and thyroid hormone concentrations in maternal and cord sera: the HOME study. Environ Res. 2020;185:109395. https://doi.org/10.1016/j.envres.2020.109395.

81. Preston EV, Webster TF, Oken E, Claus Henn B, McClean MD, Rifas-Shiman $\mathrm{SL}$, et al. Maternal plasma per- and Polyfluoroalkyl substance concentrations in early pregnancy and maternal and neonatal thyroid function in a prospective birth cohort: project viva (USA). Environ Health Perspect. 2018 126(2):027013. https://doi.org/10.1289/EHP2534.

82. Reardon AJF, Khodayari Moez E, Dinu I, Goruk S, Field CJ, Kinniburgh DW, et al. Longitudinal analysis reveals early-pregnancy associations between perfluoroalkyl sulfonates and thyroid hormone status in a Canadian prospective birth cohort. Environ Int. 2019;129:389-99. https://doi.org/10.101 6/j.envint.2019.04.023.
83. Wang Y, Rogan WJ, Chen PC, Lien GW, Chen HY, Tseng YC, et al. Association between maternal serum perfluoroalkyl substances during pregnancy and maternal and cord thyroid hormones: Taiwan maternal and infant cohort study. Environ Health Perspect. 2014;122(5):529-34. https://doi. org/10.1289/ehp.1306925.

84. Wang $Y$, Starling AP, Haug LS, Eggesbo M, Becher G, Thomsen C, et al. Association between perfluoroalkyl substances and thyroid stimulating hormone among pregnant women: a cross-sectional study. Environ Health. 2013;12(1):76. https://doi.org/10.1186/1476-069X-12-76

85. Webster GM, Venners SA, Mattman A, Martin JW. Associations between perfluoroalkyl acids (PFASs) and maternal thyroid hormones in early pregnancy: a population-based cohort study. Environ Res. 2014;133:338-47. https://doi.org/10.1016/j.envres.2014.06.012.

86. Xiao C, Grandjean P, Valvi D, Nielsen F, Jensen TK, Weihe $P$, et al. Associations of exposure to Perfluoroalkyl substances with thyroid hormone concentrations and birth size. J Clin Endocrinol Metab. 2020;105(3):735-45. https://doi.org/10.1210/clinem/dgz147.

87. Yang L, Li J, Lai J, Luan H, Cai Z, Wang Y, et al. Placental transfer of Perfluoroalkyl substances and associations with thyroid hormones: Beijing prenatal exposure study. Sci Rep. 2016;6(1):21699. https://doi.org/10.1038/srep21699.

88. Berg V, Nøst TH, Pettersen RD, Hansen S, Veyhe AS, Jorde R, et al. Persistent organic pollutants and the association with maternal and infant thyroid homeostasis: a multipollutant assessment. Environ Health Perspect. 2017; 125(1):127-33. https://doi.org/10.1289/EHP152.

89. Ballesteros V, Costa O, Iñiguez C, Fletcher T, Ballester F, Lopez-Espinosa MJ. Exposure to perfluoroalkyl substances and thyroid function in pregnant women and children: a systematic review of epidemiologic studies. Environ Int. 2017:99:15-28. https://doi.org/10.1016/j.envint.2016.10.015.

90. Hernán MA, Robins JM. Causal inference: what if. Boca Raton: Chapman \& Hall/CRC; 2020.

91. Pearl J: Causality: models, reasoning, and inference. Boston: Cambridge University Press; 2000.

92. Greenland S, Pearl J, Robins JM. Causal diagrams for epidemiologic research. Epidemiology. 1999;10(1):37-48.

93. Weinberg CR. Can DAGs clarify effect modification? Epidemiology. 2007; 18(5):569-72.

94. Hernán MA, Hernández-Díaz S, Werler MM, Mitchell AA. Causal knowledge as a prerequisite for confounding evaluation: an application to birth defects epidemiology. Am J Epidemiol. 2002;155(2):176-84. https://doi.org/10.1093/a je/155.2.176

95. Shrier I, Platt RW. Reducing bias through directed acyclic graphs. BMC Med Res Methodol. 2008;8(1):70. https://doi.org/10.1186/1471-2288-8-70.

96. Naimi Al, Cole SR, Kennedy EH. An introduction to g methods. Int J Epidemiol. 2017;46(2):756-62. https://doi.org/10.1093/ije/dyw323.

97. VanderWeele TJ, Vansteelandt S. Mediation analysis with multiple mediators. Epidemiol Methods. 2014;2(1):95-115. https://doi.org/10.1515/em-2012-0010

98. Greenland S, Robins JM. Confounding and misclassification. Am J Epidemiol. 1985;122(3):495-506. https://doi.org/10.1093/oxfordjournals.aje.a114131.

99. Conti A, Strazzeri C, Rhoden KJ. Perfluorooctane sulfonic acid, a persistent organic pollutant, inhibits iodide accumulation by thyroid follicular cells in vitro. Mol Cell Endocrinol. 2020;515:110922. https://doi.org/10.1016/j. mce.2020.110922.

100. Buckalew AR, Wang J, Murr AS, Deisenroth C, Stewart WM, Stoker TE, et al. Evaluation of potential sodium-iodide symporter (NIS) inhibitors using a secondary Fischer rat thyroid follicular cell (FRTL-5) radioactive iodide uptake (RAIU) assay. Arch Toxicol. 2020;94(3):873-85. https://doi.org/10.1007/s00204-020-02664-y.

101. Kim J, Lee G, Lee YM, Zoh KD, Choi K. Thyroid disrupting effects of perfluoroundecanoic acid and perfluorotridecanoic acid in zebrafish (Danio rerio) and rat pituitary (GH3) cell line. Chemosphere. 2021;262:128012. https://doi.org/10.1016/j.chemosphere.2020.128012.

102. Wang J, Shi G, Yao J, Sheng N, Cui R, Su Z, et al. Perfluoropolyether carboxylic acids (novel alternatives to PFOA) impair zebrafish posterior swim bladder development via thyroid hormone disruption. Environ Int. 2020;134: 105317. https://doi.org/10.1016/j.envint.2019.105317.

103. Weiss JM, Andersson PL, Lamoree MH, Leonards PE, van Leeuwen SP, Hamers T. Competitive binding of poly- and perfluorinated compounds to the thyroid hormone transport protein transthyretin. Toxicol Sci. 2009;109(2): 206-16. https://doi.org/10.1093/toxsci/kfp055.

104. Yu WG, Liu W, Jin YH. Effects of perfluorooctane sulfonate on rat thyroid hormone biosynthesis and metabolism. Environ Toxicol Chem. 2009;28(5): 990-6. https://doi.org/10.1897/08-345.1. 
105. Williams FL, Watson J, Ogston SA, Visser TJ, Hume R, Willatts P. Maternal and umbilical cord levels of T4, FT4, TSH, TPOAb, and TgAb in term infants and neurodevelopmental outcome at 5.5 years. J Clin Endocrinol Metab. 2013; 98(2):829-38. https://doi.org/10.1210/jc.2012-3572.

106. Jiang Q, Gao H, Zhang L. Metabolic Effects PFAS. In: JC DW, editor. Toxicological Effects of Perfluoroalkyl and Polyfluoroalkyl Substances. Cham: Springer International Publishing; 2015. p. 177-201.

107. Blake BE, Fenton SE. Early life exposure to per- and polyfluoroalkyl substances (PFAS) and latent health outcomes: a review including the placenta as a target tissue and possible driver of peri- and postnatal effects. Toxicology. 2020;443:152565. https://doi.org/10.1016/j.tox.2020.152565.

108. Hagenaars A, Knapen D, Meyer IJ, van der Ven K, Hoff P, De Coen W. Toxicity evaluation of perfluorooctane sulfonate (PFOS) in the liver of common carp (Cyprinus carpio). Aquat Toxicol. 2008;88(3):155-63.

109. Blake BE, Cope HA, Hall SM, Keys RD, Mahler BW, McCord J, et al. Evaluation of maternal, embryo, and placental effects in CD-1 mice following gestational exposure to Perfluorooctanoic acid (PFOA) or Hexafluoropropylene oxide dimer acid (HFPO-DA or GenX). Environ Health Perspect. 2020;128(2):27006. https://doi.org/10.1289/EHP6233.

110. Sant KE, Jacobs HM, Borofski KA, Moss JB, Timme-Laragy AR. Embryonic exposures to perfluorooctanesulfonic acid (PFOS) disrupt pancreatic organogenesis in the zebrafish, Danio rerio. Environ Pollut. 2017;220(Pt B):807-17.

111. Ojo AF, Peng C, Ng JC. Combined effects and toxicological interactions of perfluoroalkyl and polyfluoroalkyl substances mixtures in human liver cells (HepG2). Environ Pollut. 2020;263(Pt B):114182.

112. Bobb JF, Valeri L, Claus Henn B, Christiani DC, Wright RO, Mazumdar M, et al. Bayesian kernel machine regression for estimating the health effects of multi-pollutant mixtures. Biostatistics. 2015;16(3):493-508.

113. Eryasa B, Grandjean P, Nielsen F, Valvi D, Zmirou-Navier D,

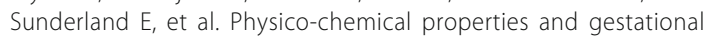
diabetes predict transplacental transfer and partitioning of perfluoroalkyl substances. Environ Int. 2019;130:104874. https://doi. org/10.1016/j.envint.2019.05.068.

114. Li X, Ye L, Ge Y, Yuan K, Zhang Y, Liang Y, et al. In utero perfluorooctane sulfonate exposure causes low body weights of fetal rats: a mechanism study. Placenta. 2016;39:125-33. https://doi.org/10.101 6/j.placenta.2016.01.010.

115. Suh CH, Cho NK, Lee CK, Lee CH, Kim DH, Kim JH, et al. Perfluorooctanoic acid-induced inhibition of placental prolactin-family hormone and fetal growth retardation in mice. Mol Cell Endocrinol. 2011;337(1-2):7-15. https:// doi.org/10.1016/j.mce.2011.01.009

116. Watkins DJ, Josson J, Elston B, Bartell SM, Shin HM, Vieira VM, et al. Exposure to perfluoroalkyl acids and markers of kidney function among children and adolescents living near a chemical plant. Environ Health Perspect. 2013; 121(5):625-30. https://doi.org/10.1289/ehp.1205838.

117. Steenland K, Fletcher T, Savitz DA. Epidemiologic evidence on the health effects of perfluorooctanoic acid (PFOA). Environ Health Perspect. 2010; 118(8):1100-8. https://doi.org/10.1289/ehp.0901827.

118. Leemans M, Couderq S, Demeneix B, Fini JB. Pesticides with potential thyroid hormone-disrupting effects: a review of recent data. Front Endocrinol (Lausanne). 2019;10:743. https://doi.org/10.3389/fendo.2019.00743.

119. Takser L, Mergler D, Baldwin M, de Grosbois S, Smargiassi A, Lafond J. Thyroid hormones in pregnancy in relation to environmental exposure to organochlorine compounds and mercury. Environ Health Perspect. 2005; 113(8):1039-45. https://doi.org/10.1289/ehp.7685.

120. Luo D, Pu Y, Tian H, Wu W, Sun X, Zhou T, et al. Association of in utero exposure to organochlorine pesticides with thyroid hormone levels in cord blood of newborns. Environ Pollut. 2017;231(Pt 1):78-86.

121. Saldana TM, Basso O, Hoppin JA, Baird DD, Knott C, Blair A, et al. Pesticide exposure and self-reported gestational diabetes mellitus in the agricultural health study. Diabetes Care. 2007:30(3):529-34. https://doi.org/10.2337/ dc06-1832.

122. Eslami B, Naddafi K, Rastkari N, Rashidi BH, Djazayeri A, Malekafzali H. Association between serum concentrations of persistent organic pollutants and gestational diabetes mellitus in primiparous women. Environ Res. 2016; 151:706-12.

123. Bedi M, von Goetz N, Ng C. Estimating polybrominated diphenyl ether (PBDE) exposure through seafood consumption in Switzerland using international food trade data. Environ Int. 2020;138:105652. https://doi.org/1 0.1016/j.envint.2020.105652.
124. Darnerud PO, Eriksen GS, Jóhannesson T, Larsen PB, Viluksela M. Polybrominated diphenyl ethers: occurrence, dietary exposure, and toxicology. Environ Health Perspect. 2001;109(Suppl 1):49-68.

125. Herbstman JB, Sjödin A, Kurzon M, Lederman SA, Jones RS, Rauh V, et al. Prenatal exposure to PBDEs and neurodevelopment. Environ Health Perspect. 2010;118(5):712-9. https://doi.org/10.1289/ehp.0901340.

126. Molehin D, Dekker Nitert M, Richard K. Prenatal exposures to multiple thyroid hormone disruptors: effects on glucose and lipid metabolism. J Thyroid Res. 2016;2016:8765049.

127. Shaffer RM, Ferguson KK, Sheppard L, James-Todd T, Butts S, Chandrasekaran S, et al. Maternal urinary phthalate metabolites in relation to gestational diabetes and glucose intolerance during pregnancy. Environ Int. 2019;123:588-96. https://doi.org/10.1016/j. envint.2018.12.021.

128. Adibi JJ, Lee MK, Naimi Al, Barrett E, Nguyen RH, Sathyanarayana S, et al. Human chorionic gonadotropin partially mediates phthalate association with male and female Anogenital distance. J Clin Endocrinol Metab. 2015; 100(9):E1216-24. https://doi.org/10.1210/jc.2015-2370.

129. Adibi JJ, Zhao Y, Zhan LV, Kapidzic M, Larocque N, Koistinen $H$, et al. An investigation of the single and combined phthalate metabolite effects on human chorionic gonadotropin expression in placental cells. Environ Health Perspect. 2017;125(10):107010. https://doi.org/10.1289/ EHP1539.

\section{Publisher's Note}

Springer Nature remains neutral with regard to jurisdictional claims in published maps and institutional affiliations.
Ready to submit your research? Choose BMC and benefit from:

- fast, convenient online submission

- thorough peer review by experienced researchers in your field

- rapid publication on acceptance

- support for research data, including large and complex data types

- gold Open Access which fosters wider collaboration and increased citations

- maximum visibility for your research: over $100 \mathrm{M}$ website views per year

At BMC, research is always in progress.

Learn more biomedcentral.com/submissions 